4 Holt, S. S., Boldt, E. A., Kaluzienski, L. J., and Serlemitsos, P. J., Nature, 256, $108(1975)$

5 Evans, W. D., Belian, R. D., and Conner, J. P., Astrophys. J. Lett., 159, L57 (1970)

6 Matilsky, T. A., Giacconi, R., Gursky, H., Kellogg, E. M., and Tananbaum, H. D., Astrophys. J. Lett., 174, L53 (1972).

7 Kaluzienski, L. J., et al., Astrophys. J. (in the press).
8 Ives, J. C., Sanford, P. W., and Bell-Burnell, S. J., Nature, 254, 578 (1975).

\section{Dynamo generation in Mercury}

OBSERVATIONS during the most recent encounter of the planet Mercury by Mariner 10 seem to have confirmed the existence of a small but intrinsic magnetic dipole of about $3 \times 10^{-3}$ gauss $R_{\mathrm{M}}{ }^{3}$, where $R_{\mathrm{M}}$ is the radius of Mercury ${ }^{1}$. This field may be small enough to have a non-dynamo explanation ${ }^{1,2}$, but it is nevertheless of interest to examine whether dynamo generation is possible for the present Mercury.

Siegfried and Solomon ${ }^{3}$ have calculated models for the interior and thermal evolution of Mercury. In these models, the cosmochemical calculations of Lewis ${ }^{4}$ were used to constrain the composition. They concluded that Mercury is most likely differentiated $^{5}$, with a $\mathrm{Fe}-\mathrm{Ni}$ core extending out to about $0.7 R_{\mathrm{M}}$, and a silicate mantle. I have tested their models for four necessary (but possibly not sufficient) conditions for magnetohydrodynamic dynamo generation.

First, dynamo generation requires that the interior be at least partially fluid, since solid-state convection is too slow for dynamo generation. In the models of Siegfried and Solomon, radioactive heat sources in the silicate mantle may be sufficient for fluidity in a region extending from about $200 \mathrm{~km}$ to about $800 \mathrm{~km}$ below the planet surface. The metallic core is either partly or entirely solid, depending on when differentiation took place.

Second, dynamo generation requires an energy source that drives a flow of the fluid relative to a rigidly rotating planet. Thermal convection seems to be the only plausible energy source, since precession is insignificant ${ }^{2}$, and the other possible sources are just as unsatisfactory for Mercury as they are for the Earth ${ }^{6}$. The Siegfried and Solomon models assume that all the radioactive heat sources are in the mantle, so that thermal convection occurs in a $600 \mathrm{~km}$ thick silicate layer, but not in the stably stratified metallic core. Other heat sources in the core (latent heat, gravitation) seem to be insufficient to drive the temperature gradient superadiabatic and initiate convection. The thermal conductivity of the metallic core is sufficiently high that convection would only occur if about half of the radioactive heat sources reside in the core. One possiblity is that Mercury has a similar fraction of ${ }^{40} \mathrm{~K}$ as Lewis ${ }^{4}$ proposes for the Earth's core. This would violate Lewis's cosmochemical arguments, since potassium compounds are too volatile to condense from the primitive solar nebula at Mercury's distance from the Sun.

Third, dynamo generation requires that the magnetic field diffusion time, $\tau_{M}$, exceeds the characteristic fluid flow (convective) time scale $\tau_{C}$. The ratio of $\tau_{M}$ to $\tau_{C}$ is known as the magnetic Reynold's number. In the convecting silicate layer, simple mixing length theory ${ }^{7}$ predicts a convective velocity of $0.1 \mathrm{~cm} \mathrm{~s}^{-1}$ for the expected heat fiux ${ }^{3}$ of about $50 \mathrm{erg} \mathrm{cm}^{-2} \mathrm{~s}^{-1}$. A similar estimate applies to the Earth's outer core ${ }^{8}$ and is unlikely to be incorrect by more than an order of magnitude. The corresponding convective time scale is $\tau_{\mathrm{c}} \sim 1-10 \mathrm{yr}$. The magnetic field diffusion time is given by ${ }^{2}$

$$
\tau_{\mathrm{M}} \approx 10^{-9} \sigma l^{2} \mathrm{~s}
$$

where $\sigma$ is the electrical conductivity in $\Omega^{-1} \mathrm{~cm}^{-1}$ and $l$ is the smallest dimension of the convection region. The requirement ${ }_{M}>\tau_{C}$ implies $\sigma>10-100 \Omega^{-1} \mathrm{~cm}^{-1}$. This would be easily satisfied in the core $\left(\sigma \sim 10^{3}\right.$ or $\left.10^{4} \Omega^{-1} \mathrm{~cm}^{-1}\right)$ but not in the miconducting silicates. In the Siegfried and Solomon models, the temperature is at most about $2,100 \mathrm{~K}$ in the mantle. At this temperature, the conductivity in the Earth is probably less than $0.1 \Omega^{-1} \mathrm{~cm}^{-1}$ (ref. 9). Cosmochemical arguments ${ }^{4}$ indicate that the Mercurian mantle should be somewhat different from the Earth, with $\mathrm{MgSiO}_{3}$ prominent, but a generous extrapolation of high temperature laboratory experiments on enstatite ${ }^{10}$, indicates that a conductivity in excess of $1 \Omega^{-1} \mathrm{~cm}^{-1}$ is very unlikely. This figure is for solid materials, but these minerals (unlike pure germanium or silicon)are not likely to change their short range order or conductivity dramatically upon melting ${ }^{11}$. (The conductivity may increase by as much as an order of magnitude, but certainly not several orders of magnitude.) I conclude that the liquid silicate conductivity is probably inadequate for dynamo generation. I note that generation in a thin silicate layer near the surface of the planet is likely to lead to a substantially non-dipolar field at the planetary surface.

Fourth, dynamo generation requires a fluid flow of sufficient complexity to satisfy Cowling's theorem ${ }^{12}$. In particular, the requirement of a non-axisymmetric field is usually interpreted to imply a need for "rapid rotation" of the planet (although other alternatives may exist ${ }^{13}$ ). Actually, "rapid rotation" only means that the Coriolis force has an important effect on convective flow. A measure of this is the ratio of inertial to Coriolis forces, $v / \Omega l$, where $v \sim 0.1 \mathrm{~cm} \mathrm{~s}^{-1}$ is a typical convective velocity in the absence of rotation, and $\Omega \sim 1 \times 10^{-6} \mathrm{~s}^{-1}$ is the planetary angular velocity. For $l \leqslant 10^{5} \mathrm{~cm}$, this ratio (which is a nominal Rossby number for the flow) is less than unity, and the Coriolis force is important. For the largest scale convective flows in Mercury, $l \approx 10^{7} \mathrm{~cm}$, so that in this respect Mercury is a rapidly rotating planet. The requirements of Cowling's theorem may then be satisfied because of the effect of planetary rotation alone.

In a more complete theory, such as that attempted by Parker ${ }^{14}$, and more recently in rigorous work by Busse (unpublished), the criterion for dynamo generation is generally more stringent than the simple ones we have discussed. A complete theory would also make a prediction for the field magnitude. A feature of the Busse theory is that if the core is convecting, then the small field of Mercury would be a consequence of the slow planetary rotation. Unlike the Earth, the ohmic dissipation in Mercury is likely to be completely insignificant compared with any important energy source such as radioactivity.

I conclude that a literal interpretation of the cosmochemical calculations of Lewis ${ }^{4}$ implies that dynamo generation in Mercury is improbable. Generation would be possible if the metallic core were contaminated with substantial amounts of radioactive material. It would then follow that not all the material that comprises Mercury condensed from the solar nebula at the present distance of Mercury from the Sun. The magnitude of the field and any future multipolar analysis (such as might be achieved by an orbiter) will be important in determining the field source.

I thank F. H. Busse, N. F. Ness, and S. C. Solomon for discussions. This work is supported by grants from NASA and the NSF.

\section{J. Stevenson}

Laboratory of Atomic and Solid State Physics,

Cornell University, Ithaca, New York 14853

Received June 9; accepted July 1, 1975.

\footnotetext{
Ness, N. F., Behannon, K. W., Lepping, R. P., and Whang, Y. C., Nature 255, 204-205 (1975).

2 Stevenson, D. J., Icarus, 22, 403-415 (1974).

Siegfried II, R. W., and Solomon, S. C., Icarus, 23, 192-205 (1974).

4 Lewis, J. S., Earth planet Sci. Lett., 15, 286-290 (1972).

Murray, B. C., et al., Science, 185, 169-178 (1974).

7 Schwarzchild, M., Structure and Evolution of Stars, 47 (Princeton University Press, Princeton, 1958)

8 Frazer, N., Geophys. J. R. astr. Soc., 34, 193-201 (1973).

9 Stacey, F. D., Physics of the Earth, 144 (Wiley, New York, 1969).

9 Stacey, F. D., Physics of the Earth, 144 (Wiley, New York, 1969).

11 Gubanov, A. I Quantum Electron Theory of Amorphous Conductors, (Consuitants Bureau, New York, 1965)

12 Cowling, T. A., Mon. Not. R. astr. Soc., 94, 39-48 (1934).

13 Busse, F. H., J. fluid. Mech., 57, 529-544 (1973).
}

14 Parker, E. N., Astrophys. J., 164, 491-509 (1971). 